Slavica

bruxellensia

\section{Slavica bruxellensia}

Revue polyphonique de littérature, culture et histoire

slaves

$6 \mid 2010$

Linguistique russe

\title{
Altérité slave et imaginaire de la langue.
}

Quelques cas de figures...

\section{Cécile Gauthier}

\section{(2) OpenEdition}

Journals

Édition électronique

URL : http://journals.openedition.org/slavica/340

DOI : 10.4000/slavica.340

ISSN : 2034-6395

Éditeur

Université libre de Bruxelles - ULB

Édition imprimée

Pagination : 6-18

ISSN : 2031-7654

Référence électronique

Cécile Gauthier, «Altérité slave et imaginaire de la langue. », Slavica bruxellensia [En ligne], 6 | 2010, mis en ligne le 15 juin 2010, consulté le 21 avril 2019. URL : http://journals.openedition.org/slavica/340 ; DOI : $10.4000 /$ slavica.340

Ce document a été généré automatiquement le 21 avril 2019

\section{(c) $(1) \odot$}

Les contenus de Slavica bruxellensia sont mis à disposition selon les termes de la Licence Creative Commons Attribution - Pas d'Utilisation Commerciale - Pas de Modification 3.0 France. 


\title{
Altérité slave et imaginaire de la langue.
} Quelques cas de figures...

\author{
Cécile Gauthier
}

1 La langue joue un rôle décisif dans les questionnements sur l'identité : on tend en effet (tel est le postulat du concept «d'identité linguistique ») à enraciner l'identité dans la langue, cette dernière étant abusivement conçue comme un objet homogène et appropriable. L'intrication de la langue et de la culture présente des racines et des prolongements nationaux, voire nationalistes, et on sait quel rôle capital elle a joué dans le cadre de l'éveil des nationalités en Europe centrale et orientale. Dans la mesure où identité et altérité constituent les deux facettes d'un même objet, on peut inversement supposer que « la langue de l'autre » est perçue comme un des éléments déterminants de sa différence, suscitant toutes sortes de jugements, de rêveries et de fantasmes. Notre angle d'étude est donc celui de "l'imaginaire de la langue », qui emprunte son exigence de déconstruction des idéologies aux travaux imagologiques ${ }^{1}$ inspirés par le postcolonialisme, ainsi qu'aux recherches sur la «linguistique spontanée $»^{2}$. Mais notre proposition consiste à creuser, à la croisée de plusieurs disciplines, un sillon propre aux recherches comparatistes, en recentrant la réflexion sur la langue, en tant qu'elle constitue l'objet de la réflexion, mais également qu'elle fonde une méthode, l'étude à partir du mot $^{3}$.

2 Ainsi c'est en adoptant cet angle d'étude, «l'imaginaire de la langue », que nous nous proposons de considérer l'altérité slave telle qu'elle se donne à lire dans un corpus de textes de langue française et allemande. Postulant une correspondance entre l'écriture de "l'étranger » et celle de sa langue, nous analyserons les discours tenus sur les langues slaves, et ce qu'ils révèlent en termes de représentations de l'altérité slave, dans un corpus double, lexicographique et romanesque ${ }^{4}$. Nous présenterons de façon successive les discours transparaissant dans les dictionnaires et encyclopédies, puis dans les romans, tout en insistant sur la continuité d'un corpus à l'autre. 


\section{Le discours encyclopédique}

3 Il faut commencer par rappeler dans quel contexte épistémologique se situe le discours sur les langues au XIX ${ }^{e}$ siècle. Le parallèle que nous postulons entre les représentations du locuteur et de sa langue est en effet déjà décelable dans les encyclopédies du XVIII ${ }^{\mathrm{e}}$ siècle, mais l'articulation se précise et se systématise au siècle suivant, lors du développement de la linguistique comparée, qui repose sur une mise en comparaison de diverses "familles de langues", au nombre desquelles les langues slaves, jusqu'ici inégalement connues et étudiées ${ }^{5}$. C'est précisément au cours des premières décennies du XIX ${ }^{e}$ siècle qu'est «ressuscité » le mot slave, qui avait presque disparu des langues française et allemande dans une acception contemporaine ${ }^{6}$, et qui accompagne la création du groupe des « Slaves ». Le nom de la langue s'avère donc créateur d'ontologie, comme le démontre le linguiste Patrick Sériot? ${ }^{7}$. La "révolution» provoquée par l'essor de la linguistique comparée conduit à une articulation sans précédent entre peuple et langue. Le ressort des recherches passionnées des philologues est la quête de la langue-mère, ou Ursprache : à partir de l'étude du sanskrit et de la comparaison entre diverses familles de langues, se trouve posée l'hypothèse de l'existence d'une langue originelle indoeuropéenne, commune à la majorité des langues parlées en Europe, qualifiées de « langues-filles ». Le lien entre langue et « race » est progressivement théorisé, et de la rêverie associée à une langue-mère, d'aucuns en viennent à rêver à un peuple originel, supérieur, les Aryens, dont certains peuples d'Europe descendraient plus directement que d'autres. À partir du moment où la classification des langues se mêle à un essai de hiérarchisation des peuples, des dérives « racialistes » émergent, qui se durcissent dans la seconde moitié du XIX siècle pour se déchaîner au siècle suivant.

Dans un tel contexte, l'évocation des langues slaves, ou de «la langue slave », évoquée au singulier, se fait plus précise. Deux traits sont particulièrement soulignés: l'étendue considérable de l'espace linguistique que ces langues déterminent, et leur parenté avec le sanskrit, qui en fait des idiomes "primitifs». Ces traits sont mentionnés avec un étonnement quelque peu fasciné, parfois accompagnés d'un geste de dévaluation révélateur d'une certaine méfiance. La première remarque, récurrente dans les articles lexicographiques du XIX siècle, se rencontre déjà dans les ouvrages du siècle précédent, ainsi dans l'Encyclopédie de Diderot et d'Alembert (1751-1765) : «L'esclavon passe pour être, après l'arabe, la langue la plus répandue depuis la mer Adriatique jusqu'à la mer du Nord, et depuis la mer Caspienne jusqu'à la mer Baltique " ${ }^{8}$. L'évocation devient spectaculaire dans les ouvrages du XIX ${ }^{\mathrm{e}}$ siècle (qui entérinent la création de l'unité des Slaves), ainsi dans le Conversations-Lexikon Brockhaus en douze volumes (1836) :

5 L'imaginaire géographique du lecteur est mis en éveil par la mention de ces terres éloignées aux noms évocateurs, tels que le Kamtchatka. C'est encore davantage le cas dans le Grand Dictionnaire universel du XIXe siècle de Pierre Larousse (1875):

Les idiomes slaves occupent en Europe un espace plus étendu que toute autre langue. Depuis la Dwina, à l'est, jusqu'aux monts des Métaux (Bohême et Saxe), à l'ouest, et, dans les anciens temps encore, bien plus loin vers le cœur même de l'Allemagne; depuis les rives de la mer Glaciale jusqu'à celles de la mer Adriatique et de la mer Noire et jusqu'à l'Archipel grec, voilà l'énorme domaine des Slaves d'Europe. Leur langue s'est aussi propagée à travers l'Asie septentrionale ou la Sibérie jusqu'au nord de l'Amérique, en sorte qu'elle occupe un sixième de la surface habitable de tout le globe. ${ }^{10}$ 
Pierre Larousse est un fervent défenseur des peuples slaves, et ce d'autant plus qu'ils peuvent constituer un allié de poids face à l'ennemi allemand. Aussi cette description a-telle vocation à susciter chez le lecteur une admiration émerveillée, mais on entrevoit comment, dans un autre contexte, cette « propagation de la langue » pourrait éveiller des images de déferlement ou d'invasion.

7 Pour ce qui est de la parenté supposée des langues slaves avec le sanskrit, elle en fait $a$ priori des langues proches de la source originelle, et, par là même, dignes de la plus grande estime. Cette parenté est discutée au cours du XIX ${ }^{e}$ siècle, et progressivement remise en question, en particulier par les érudits allemands ${ }^{11}$, souvent eux-mêmes désireux de prouver la parenté de leur propre langue avec le sanskrit. Quoi qu'il en soit, la 《richesse antique ${ }^{12}$ des langues slaves est unanimement célébrée par les lexicographes germaniques et français. On lit ainsi dans le Conversations-Lexikon Brockhaus (1836) :

Les spécificités ici présentées comptent de fait au nombre des critères d'excellence d'un idiome, puisqu'elles dénotent un caractère synthétique qui permet une expression plus précise sans compliquer la structure syntaxique de la phrase. Larousse, se réclamant d'August Schleicher, célèbre pour sa part les langues slaves, leur « puissante vitalité dans le verbe et dans le substantif », leur "force productive qui est infiniment supérieure à celle [des] idiomes occidentaux décrépits ». Mais si cette vitalité primitive est souvent interprétée comme gage de pureté, une autre lecture, sans doute héritée des siècles précédents, est toujours prête à refaire surface: alors que la nature mélodieuse des langues slaves est fréquemment évoquée, un certain nombre de critiques pointent à l'inverse le caractère en apparence non-policé des langues slaves, signe de barbarie plutôt que de perfection originelle. Louis-Nicolas Bescherelle, à l'article russe, précise par exemple que « russe s'emploie quelquefois en mauvaise part, pour désigner la barbarie dans le langage $»^{14}$, citant le quatrain suivant (anonyme) :

9 L'impression de dureté et de rudesse mise en avant dans nombre d'articles provient en grande partie d'une perception graphique de la langue, fondée sur la prédominance " exotique » des consonnes dans nombre de langues slaves ${ }^{15}$. Ce trait est souvent présenté comme rebutant, peut-être jugé révélateur de l'absence de règles appliquées à la stabilisation de la langue dans sa forme écrite. Or cette stabilisation serait perçue, particulièrement en France, comme la marque d'une civilisation avancée et supérieure. Ainsi l'admiration pour la " primitivité » est sans cesse susceptible de se retourner en son contraire, conférant aux langues slaves une place ambivalente au sein de la famille indoeuropéenne: valorisées car supposément proches des origines, ces langues sont aussi considérées (de même que leurs locuteurs, qui seraient restés extérieurs à la marche de l'Histoire) comme moins évoluées que les langues d'Europe occidentale.

\section{Le discours romanesque}

10 Dans les romans, une des métaphores par lesquelles les langues slaves sont souvent désignées est celle du chant. Cette métaphore est en soi relativement courante pour désigner une langue jugée mélodieuse, en particulier lorsqu'il s'agit d'une langue étrangère, inconnue, perçue comme un flux dépourvu de signification ${ }^{16}$. Mais, à la lumière des analyses précédemment développées, il apparait que cette métaphore détient un sens particulier pour ce qui est des «Slaves », considérés, parmi les Européens, comme des "primitifs de l'intérieur». Leur chant apparaît aussi bien comme le mode 
d'expression brut de l'être "peu civilisé ", que comme une manifestation artistique, la définition même du " primitif » résidant dans cette conciliation entre art et nature. Nous évoquerons successivement trois catégories de chant, chacun correspondant à une perception du fantasmatique "être slave »: le chant du peuple, dépositaire d'une âme ancestrale paysanne ; le chant séduisant et séducteur du Slave objet du désir; et le chant de guerre du « Slave esclave » révolté.

\subsection{Le chant du peuple}

11 Les représentants de la slavité sont dans bon nombre de romans, en particulier dans le corpus germanique, des figures $d u$ "petit peuple $»^{17}$. Leurs modes d'expressions apparaissent peu élaborés, comme s'ils n'avaient pas totalement accédé à un langage articulé et rationnel. Beaucoup d'entre eux sont ainsi silencieux, à la limite du mutisme. Ou encore, tel le Croate Zwonimir, dans Hôtel Savoy de Joseph Roth, ils s'expriment avec leur corps, de façon très brute ${ }^{18}$ :

C'est un langage du corps, immédiat et prosaïque, donné à voir plus qu'à entendre. Ces évocations de personnages obstinément muets ou se livrant à de grotesques gesticulations pourraient traduire une forme de condescendance de l'homme "cultivé » envers «l'homme slave ", jugé moins évolué, et recouper la représentation courante de ces peuples comme des peuples passifs, inférieurs, immobilisés dans un temps immémorial. Mais, comme le montre la citation de Roth, une admiration réelle se fait jour pour une forme d'expression valorisée comme plus efficace et, surtout plus authentique, moins mensongère. Au-delà de l'amusement indulgent que suscite le personnage de Zwonimir, il y a de fait une incontestable supériorité de son mode d'être, de son « sens de la communauté ", qui lui permet de fraterniser avec les autres hommes. Le narrateur envie son "sang paysan", synonyme d'une proximité avec la nature et d'une homogénéité que lui, en tant qu'homme moderne, a perdue. Cela se joue sur le plan du langage : ainsi, lorsque le narrateur s'accuse lui-même, non sans une certaine coquetterie, d'être un égoïste, Zwonimir met en cause cette maitrise artificielle de la langue.

On reconnaît là un topos important au $\mathrm{XX}^{\mathrm{e}}$ siècle, qui est la crise de la culture, rendue manifeste dans la crise du langage. Les langues du monde "occidental ", usées et chargées d'artifice, se prêtent aisément au mensonge, alors que les langues dites "primitives» telles que les langues slaves, plus spontanées et proches des origines, permettent l'expression de la vérité. Là n'est pas la moindre supériorité du "primitif »: cette vérité touche en effet à l'art, et ce n'est pas un hasard si «l'âme slave ", tellement insaisissable, se donne à entrevoir dans le chant présenté comme un prolongement « naturel » des langues slaves.

Le chant, qui se situe entre manifestation esthétique élaborée et expression spontanée d'un locuteur "enfant", révèle l'âme du peuple. Dans les romans, l'âme slave est fréquemment mise en scène de façon codifiée, selon un schème narratif récurrent : un personnage spectateur, et avec lui le lecteur, assistent au dévoilement, par un chant mélancolique et doux, de l'intimité et de la spiritualité d'une âme. Dans plus d'un roman, un(e) Russe est présenté(e) en train de chanter d'une façon si déchirante qu'il/elle suscite les larmes de l'auditoire, preuve ultime de l'authenticité de l'émotion exprimée. On le voit dans Rouletabille chez le tsar, roman dans lequel Gaston Leroux multiplie avec facétie les clichés sur la culture russe. La jeune Natacha chante une « lente mélopée », accompagnée par un musicien jouant de la guzla, dont il tire des « sons plaintifs » : 
Pour ce qui est des Slaves d'Europe centrale, davantage présents dans le corpus germanique, leur chant est souvent présenté comme un chant collectif, témoignant d'un folklore vivace et d'une continuité avec les ancestrales croyances et traditions paysannes, même au cœur de la cité. Ainsi, dans le roman de Max Brod ${ }^{22}$, Une servante tchèque (1909), toute la ville de Prague retentit d'un chant rural. Le narrateur, un jeune Viennois, pousse la porte d'un intérieur tchèque, sans y être invité, et se trouve subrepticement témoin d'une scène de l'intimité "nationale $»^{23}$. Autour d'une table, des femmes chantent en chœur un air de la campagne, que le jeune homme reconnaît immédiatement :

La caractéristique de ce chant, outre sa mélancolie, réside dans son "rythme », élément porteur de slavité - vraisemblablement une certaine lenteur, trait récurrent de la gravité des musiques slaves dans les textes étudiés. Cet air de la campagne constitue un leitmotiv dans le roman. Lorsque le personnage, épris d'une jeune servante tchèque, consomme enfin son union avec elle, le don du corps est consacré par le don de la voix, qui est aussi communion avec tout un peuple :

17 Cet exemple révèle que le chant, s'il est voie d'accès à «l'âme slave " et s'il manifeste l'unité d'un peuple et d'une culture, est aussi, pour l'observateur extérieur, le vecteur du désir : désir de l'étranger aussi bien que de la langue étrangère.

\subsection{La séduction du chant}

De fait, le motif du chant, susceptible de susciter un désir sensuel, constitue un des éléments déterminants du pouvoir de séduction exercé par les Slaves, et rejoint un archétype fameux, celui du chant des sirènes. Celui-ci subjugue et vainc les résistances de l'auditoire : les hommes envoûtés abdiquent leur volonté et leur liberté, se livrant à une mort certaine. La voix est donc porteuse d'une charge érotique : les «voix slaves» en particulier constituent un objet de désir, notamment lorsqu'elles se donnent à entendre sans que la vue soit sollicitée - montrant que «l'âme slave ", indéniablement empreinte de spiritualité, n'est pas toujours si éthérée qu'on pourrait le croire. La voix est promesse de la chair, et c'est une attirance pour le corps qui se manifeste à travers la fascination pour la voix. Sous la plume de Jean Lorrain, sulfureux représentant de la " décadence » fin-de-siècle, ces évocations se doublent du thème de la confusion des genres, et suggèrent la nature transgressive du désir. Dans le roman Très russe, les voix successives du petit groom, Petchorine (" une voix chantante et un peu grave », une "voix jeune et rauque ${ }^{26}$ ) et de la femme de chambre ( la même voix chantante et gutturale qu'on eût prise pour l'écho de celle de Petchorine») se superposent de façon troublante et déstabilisante à celle de Mme Livitinof, incarnation de la « femme slave » :

19 Au-delà de la volupté de la « voix chantante », c'est, plus fondamentalement, la langue de l'autre qui séduit. La langue slave (dans ses différentes formes) est fréquemment qualifiée de langue "douce», " harmonieuse », " mélodieuse». Mais surtout, étant une langue étrangère, elle éveille un désir de nature exotique. Elle suscite en effet une attirance en tant qu'elle est une preuve tangible de l'inconnu incompréhensible, peut-être intraduisible, et également en tant qu'elle «matérialise » le désir, par sa signifiance qui peut être métaphore du corps désiré. Cela est particulièrement visible lorsque des mots étrangers ${ }^{28}$ sont inscrits dans le texte, tels des incarnations du corps désirable de l'autre, ainsi dans le roman de Jean Lorrain précédemment cité : 
20 La question du sens, évoquée ici dans le thème de la traduction, se trouve au cœur du désir exotique, dont le caractère essentiellement paradoxal est formulé par Roland Barthes en ces termes : « Le rêve : connaître une langue étrangère (étrange) et cependant ne pas la comprendre $»^{30}$. Le caractère incompréhensible de la langue étrangère l'arrache à la contingence et au prosaïsme, et permet de nourrir les aspirations à l'idéal, spirituel et/ou charnel. Dans La Mort à Venise de Thomas Mann, la langue polonaise, incomprise, est élevée à l'expression de la pure beauté. Elle peut passer pour musique, car l'attention est portée à sa seule sonorité, ainsi lorsque Tadzio rapporte à sa famille les découvertes faites sur la plage :

21 Le désir suppose un mouvement en direction de l'objet convoité, qui peut être la familiarisation avec la langue de l'autre (par l'apprentissage), ou encore la traduction. C'est là que se noue la nature paradoxale de l'exotisme : ces gestes, donnant un accès au sens, menacent du même coup de réduire à néant le mystère originel, et donc d'annuler le désir à sa source. Le maintien du désir nécessite donc le respect d'un équilibre incertain entre distance et proximité, qui correspond précisément au souhait formulé par Barthes, celui de goûter la différence sans l'abolir.

La distance est souvent maintenue par l'existence implicite d'une barrière à ne pas franchir entre l'autre et soi. La séduction indéniable exercée par l'obscur idiome est insidieusement menaçante, et cela d'autant plus qu'il émane de la "femme fatale », qui prend plus d'une fois les traits d'une femme slave dans les romans étudiés. C'est le cas dans La Montagne magique de Mann: Hans Castorp s'éprend de Mme Chauchat, et son cousin Joachim de Maroussia, laquelle ne parle que le russe. De façon symptomatique, Joachim s'est lancé dans l'étude assidue de cette langue ${ }^{32}$ : il succombe donc au charme dangereux de la femme autant que de la langue. Si l'on revient sur les qualifications prêtées par Mann aux langues slaves, et en particulier à la langue russe, l'adjectif weich, "doux ", «souple", "suave ", recoupe des connotations inquiétantes, exprimées plus franchement par d'autres adjectifs : verwaschen, «indécis », "imprécis ", "vague », ou encore knochenlos, « désossé $»^{33}$. La langue slave, « idiome oriental» ( «das östliche Idiom » ${ }^{34}$ ) confinant à la barbarie, est dépourvue de structure et de fermeté, sournoisement liquide et instable: elle incarne le risque de l'inarticulé, la perte de la forme et, à terme, l'engloutissement dans le néant - fût-il éminemment désirable, comme c'est le cas, également, dans La Mort à Venise. Le désir de transgression, nourri par le besoin de rejoindre l'être aimé sur son territoire, qui est aussi un territoire linguistique, est mortifère : il conduit à la perte de soi, parce qu'il menace d'effacer les frontières entre le même et l'autre, entre l'identité et l'altérité.

\subsection{Le chant de guerre}

23 La langue étrangère si désirable peut donc devenir, du fait même de cet insidieux pouvoir de séduction, une menace. C'est pourquoi, en ce début de $\mathrm{XX}^{\mathrm{e}}$ siècle, les nationalistes farouches et autres tenants de la race (ancrée dans une conception essentialiste de la langue) réaffirment le maintien de frontières délimitant les objets-langues. Ces limites à ne pas franchir sont censées garantir une homogénéité synonyme d'une utopique pureté. Ainsi, la valorisation de la différence, qui est constitutive de la perception exotique, peut également se comprendre comme la manière la plus sûre de maintenir la distance, d'éviter les contacts et les possibles confusions. L'adoption, même provisoire, de la langue de l'autre, est suspecte de trahison, puisqu'elle peut être le préalable à une " conversion » 
effective à la langue, la culture, et donc la nation, de l'autre. Cela est surtout marqué dans les romans germaniques dont l'action est située dans l'Europe centrale multiculturelle, en particulier dans certains territoires où se joue une bataille entre les langues, par exemple dans la région des Sudètes ou encore en Poznanie. La langue n'échappe pas à la politisation et à une possible instrumentalisation.

Si la langue est politisée, c'est tout d'abord qu'elle accompagne un mouvement de revendication et fait entendre une voix auparavant étouffée. Nous avons déjà mentionné le fait que le Slave était souvent décrit comme silencieux, voire mutique, et ce parce qu'il incarne une figure du primitif. Mais c'est également que sa parole est muselée, censurée, par exemple en Russie, fréquemment représentée comme un empire de serfs ${ }^{35}$ où s'exerce l'oppression. Le silence du Slave doit donc être compris comme celui d'un être " dominé ». Il faut à cet égard rappeler le lien inextricable qui unit les histoires des mots slave et esclave ${ }^{36}$, et qui est prolongé dans un imaginaire de la servitude couramment associé aux représentations de la slavité. Les Slaves peuvent être décrits de façon condescendante comme des esclaves par nature, des peuples inférieurs voués à être soumis à la tutelle de peuples plus évolués. Mais ce motif peut inversement susciter la compassion, voire l'indignation, jusqu'à être mis progressivement au service d'une rhétorique plus révolutionnaire. Le roman de Leroux Rouletabille chez le tsar ${ }^{37}$ est ainsi porteur (jusqu'à un certain point) d'un regard critique à l'encontre des injustices sociales et de l'absence de liberté dans l'Empire russe. Le reporter Rouletabille traverse au petit matin la campagne russe, qu'il juge morne et désolée. Il ne peut s'empêcher de comparer « le joyeux réveil des campagnes de France » avec ce spectacle de deuil.

Ce monde réduit à l'état de mort-vivant, aveugle et privé de la parole, est soumis à ce qu'il faut reconnaître, même si cela n'est pas nommément désigné, comme une forme de censure. L'accusation porte d'autant plus que le lieu est loin d'être anodin : la petite ville en question n'est autre que Tsarkoïe Selo, résidence du tsar...

Dans le pesant univers des Slaves, seules s'élèvent quelques complaintes qui constituent l'unique mode d'expression accessible à ces peuples privés de liberté. Ces chants peuvent sembler dérisoires, voire condamnables : de fait la plainte, pour mélodieuse qu'elle soit, n'en est pas moins susceptible d'être considérée comme un gémissement fort peu viril, symptomatique non pas d'une impuissance réelle mais d'une scandaleuse tendance à la résignation (marque du supposé "fatalisme slave", qui expliquerait - et justifierait cette condition "d'esclaves»). Mais, considérés sous un autre angle, ces chants sont une des premières tentatives pour rompre le silence et formuler un discours articulé et conscient. Le chant détient une dimension subversive en puissance, par l'insatisfaction qu'il permet de formuler ${ }^{39}$ : ce mode d'expression privilégié des Slaves possède donc plus d'une facette. Il est, comme nous l'avons vu, élément folklorique, il déploie une esthétique, révèle une spiritualité, mais il s'avère également un potentiel outil politique, un moyen contourné d'affirmer peu à peu une existence contestée. Le doux chant de la campagne s'élève progressivement au cri de révolte. Dans une des scènes centrales du roman de l'Autrichien Fritz Mauthner, Der letzte Deutsche von Blatna (Le Dernier Allemand de Blatna), une procession célébrant la victoire politique des Tchèques dans les Sudètes dégénère en un assaut de violence primaire contre les Allemands. Les mélancoliques airs slaves laissent place au chant national, avant de se métamorphoser en un chant de mutinerie ("Trotzlied»), ponctué du cri «Slawa!», au message on ne peut plus radical: «Mort et damnation à tous les ennemis!» («Tod und Hölle allen Feinden!» ${ }^{40}$ ). En cette veille de Première Guerre mondiale, le chant slave n'est plus l'expression romantique de 
l'âme du peuple, mais le cri virulent d'une nation qui veut accéder à une reconnaissance historique et politique, menaçant par là la langue - et le corps - des Allemands avec qui elle partage ce territoire frontalier.

Dans le corpus de langue française, où dominent les évocations de la Russie, ce n'est pas un chant de combat national qui domine, mais celui de la Révolution, là aussi mis en scène de façon spectaculaire ${ }^{41}$. C'est le cas dans la description que fait Joseph Kessel de l'entrée dans la ville des bolcheviks, juchés sur des camions :

Le chant est une manifestation de la liberté conquise ainsi que de la rage contenue qui éclate enfin : il est donc une célébration de ce renversement de situation par laquelle l'esclave a pris la place du maître. Mais il constitue également en soi, comme le suggère la juxtaposition des verbes "vouloir", "chanter", et "tuer", un véritable acte révolutionnaire. Il a notamment un pouvoir d'entraînement fascinant et quasi magnétique.

29 Ainsi le chant de révolte des Slaves, qui se donne à entendre dans des contextes politiques très divers, est néanmoins presque toujours tendu entre deux postulats: sa violence intrinsèque est susceptible de le faire régresser dans le domaine du non articulé, du non verbal, stigmate de bestialité. Mais le mouvement peut aussi bien être inverse : le cri peut en effet être un aboutissement, un avènement, le premier pas vers une parole construite, mûre, celle d'un peuple entrant dans l'Histoire. L'apprentissage de la parole est la métaphore de l'accession du peuple enfant à l'âge adulte, à l'émancipation politique et la reconnaissance de sa langue. Par ailleurs cette prise de parole, si elle est d'une rare violence, fait apparaître une autre facette de l'esclave : son soulèvement est espoir de libération à l'échelle de l'humanité. Le Slave esclave est un libérateur à la stature universelle, pourvu du don de fraternité, d'humanité. En prenant sa place et en faisant résonner sa langue dans le concert des nations, il apporte un accomplissement au devenir de l'Homme.

30 La lecture croisée des deux corpus, lexicographique et romanesque, a permis de mettre en lumière l'existence d'un continuum d'un type de discours à un autre, révélant que, dans la construction et la circulation du savoir, et des représentations qui en découlent, les frontières sont plus poreuses qu'on ne pourrait et voudrait le croire entre dictionnaire et roman. Le dictionnaire n'est pas le lieu d'une langue neutre, objective et indiscutable, pas plus que la littérature, à l'inverse, n'échappe totalement à l'esprit du temps, qui marque la langue. Dans le cas qui nous intéresse, on voit que l'ambivalence des discours tenus sur les langues et les locuteurs slaves dans le corpus lexicographique trouve un écho dans le balancement des représentations dans les romans, montrant combien la construction de l'altérité repose sur la réversibilité des images. Ainsi, dans l'esquisse de typologie proposée, il y a continuité d'une catégorie de chant à une autre, mais il y a aussi continuité du discours encyclopédique au discours romanesque, et ce aussi bien pour ce qui est du contenu déployé que de la réversibilité de ce contenu. À la figure ambiguë du Slave, cet "autre intérieur", proche et lointain, miroir et repoussoir, effrayant et désirable, correspond le jugement sur des langues elles aussi conçues comme primitives, et pour cette raison à la fois admirées comme les plus proches de la perfection originelle, et décriées comme les moins évoluées, encore brutes, voire barbares. 


\section{NOTES}

1. Pour une synthèse sur cette question, nous renvoyons aux travaux de Daniel-Henri Pageaux : Littératures et cultures en dialogue, L'Harmattan, Paris,2007, 338 p. \& La Littérature générale et comparée, Colin, Paris, 1994, $191 \mathrm{p}$.

2. L'étude des représentations des langues, en particulier telles qu'elles transparaissent dans les discours épilinguistiques, constitue un champ d'étude propre de la linguistique. Le slaviste Patrick Sériot parle de « linguistique spontanée » (Voir «La linguistique spontanée des traceurs de frontières ", in : Langue et nation en Europe centrale et orientale du XVIII ${ }^{e}$ siècle à nos jours (Sous la direction de Sériot P.), in : « CAHIERS DE L'ILSL ", n 8, Lausanne, 1996, pp. 277-304), qu'il conçoit comme une variante de ce que Sylvain Auroux a appelé la « linguistique fantastique » (Auroux $\mathrm{S}$. et alii, La linguistique fantastique, Joseph Clims Éditeur, Denoël, Paris, 1985, 380 p.). Anne-Marie Houdebine-Gravaud a quant à elle proposé le concept « d'Imaginaire linguistique » (L'Imaginaire linguistique, L'Harmattan, Paris, 2002, 153 p.). Nous abordons pour notre part dans cet article l'imaginaire de la langue spécifiquement en lien avec la notion d'altérité : il ne s'agit pas en effet de l'appréciation portée par le locuteur sur sa propre langue, encore que l'étude des transferts culturels montre un constant va-et-vient et une fécondation réciproques entre "auto » et «hétéro-représentations ».Les discours «occidentaux» sur les langues slaves héritent donc, jusqu'à un certain point, des discours produits par les « Slaves » eux-mêmes.

3. Cette méthode consiste à partir des mots, porteurs d'une histoire faite de conflits et de rêveries, vecteurs de transferts culturels, pour déployer l'imaginaire déposé dans la langue. Dans notre thèse en littérature comparée (soutenue en 2009 à l'Université Paris VIII, sous la direction de Tiphaine Samoyault), nous avons retracé l'imaginaire du mot "slave» dans les langues française et allemande. Ce mot, en tant que nom de l'autre et de sa langue, est porteur d'enjeux identitaires cruciaux.

4. Nous avons consulté dans le cadre de notre thèse plusieurs dizaines de dictionnaires et encyclopédies de langue française et allemande (XVIII $-\mathrm{XX}^{\mathrm{e}}$ siècles), ainsi qu'une cinquantaine de romans (années 1880-1930). Les bornes chronologiques du corpus romanesque, assez largement déterminées par l'Histoire et son impact sur les transferts culturels (visibilité grandissante des "peuples slaves", et fascination pour les «choses russes», notamment lorsqu'éclate la Révolution russe; crispations de plus en plus violentes des nationalismes et de la pensée racialiste au tournant du siècle, cristallisées lors de la Première Guerre mondiale), conduisent à retracer, par ce "récit du Slave », un pan de l'histoire européenne déposée dans la langue et son imaginaire. L'établissement $d u$ corpus a été établi en fonction du critère très spécifique de recherche des occurrences du mot slave. Nous avons veillé à ce que le corpus soit le plus équilibré possible d'une aire linguistique à l'autre, et représentatif de la diversité des formes littéraires de l'époque. Nous avons notamment mêlé littérature lettrée et littérature populaire, auteurs reconnus et écrivains tombés dans l'oubli. Citons quelques noms familiers : Jean Lorrain, Paul Bourget, Eugène Melchior de Vogüé, Jules Verne, Gaston Leroux, Joseph Delteil, Joseph Kessel, Romain Rolland, René Crevel; Thomas Mann, Reiner Maria Rilke, Hermann Broch, Fritz Mauthner, Leopold von Sacher-Masoch, Franz Werfel, Max Brod. Nous n'illustrerons ici notre propos qu'à l'aide d'un nombre d'exemples restreints, mais en tenant compte de l'étude du corpus dans son ensemble.

5. Alors que l'orientaliste anglais William Jones avait laissé de côté les langues slaves lorsqu'il établissait ses premières hypothèses, l'Allemand Franz Bopp, considéré comme un des fondateurs de la grammaire comparée, les intègre en 1833 à l'arbre indo-européen. Elles sont donc, tout au 
long du XIX siècle, l'objet d'un discours savant de plus en plus précis, qui trouve des répercussions également auprès d'un plus large public.

6. Il était en effet utilisé pour désigner une peuplade antique. Dans un sens contemporain, on utilisait plus volontiers le terme "esclavon », qui pouvait désigner les peuples de langue slave dans leur ensemble, mais plus souvent uniquement les Slaves du Sud, habitant l'Esclavonie (ou Slavonie, région orientale de la Croatie comprise entre la Save, la Drave et le Danube).

7. - Cf. « La linguistique spontanée des traceurs de frontières », Art. cit.

8. Encyclopédie, ou Dictionnaire raisonné des sciences, des arts et des métiers. Tome cinquième, par Denis Diderot et Jean Le Rond D'Alembert, 1751-1765, article « Esclavon », p. 943.

9. Allgemeine deutsche Real-Encyclopädie für die gebildeten Stände. Conversations-Lexikon, in zwölf Bänden, Brockhaus, Zehnter Band, Leipzig, 1836, pp.312-313. Traduction par l'auteur de cet article.

10. - Larousse P., Grand Dictionnaire universel du XIX $X^{e}$ siècle, tome quatorzième, $1^{\text {ère }}$ partie, 1875, article « Slave », p. 782.

11. Rappelons que les universités allemandes jouent un rôle précurseur dans le développement de la grammaire comparée, et sont les plus avancées dans le domaine de la slavistique.

12. - Larousse P., Op. cit., p. 783.

13. Allgemeine deutsche Real-Encyclopädie..., Op. cit., p. 313.

14. Le Dictionnaire national, ou Dictionnaire universel de la langue française, par Louis-Nicolas Bescherelle, $2^{\mathrm{e}}$ édition, 1853, p. 1237.

15. On trouve cette critique ironique sous la plume d'Honoré de Balzac, dans La Fausse maîtresse (1842) : «Qu'il soit permis d'écrire les noms comme ils se prononcent, pour épargner au lecteur l'aspect des fortifications de consonnes par lesquelles la langue slave protège ses voyelles, sans doute afin de ne pas les perdre, vu leur petit nombre.", in : La Comédie humaine II. Études de mœurs : scènes de la vie privée, Gallimard, coll. « Pléiade », Paris, 1996, p. 195.

16. Cette incompréhensibilité de la langue est bien évidemment réversible et relative : rappelons, pour ce qui touche aux relations germano-slaves, qu'en vieux slave les Allemands sont appelés « Nĕmcy », c'est-à-dire les « muets », ceux qui ne parlent pas.

17. On connaît l'importance des figures de paysans (notamment des moujik, popularisés dans la littérature russe) : ils sont présents dans les romans étudiés, ainsi dans Cours russes de Vogüé (1893), Rouletabille chez le tsar de Leroux (1913), La steppe rouge de Kessel (1923), les nouvelles galiciennes de Sacher-Masoch (années 1880), Les Désarrois de l'élève Törless de Robert Musil (1906), Le dernier Allemand de Blatna de Mauthner (1913)... On trouve également, en particulier dans le corpus autrichien, des figures de domestiques et de nourrices, par exemple dans La Marche de Radetzky de Joseph Roth (1932), Une servante tchèque de Brod (1909), Les Somnambules de Broch (1931), Barbara ou la piété de Werfel (1929)...

18. L'expressivité du corps peut précisément se combiner avec l'absence de prise de parole: Barbara, la nourrice du roman de Werfel, ne parle quasiment pas, mais sa présence est indissociable d'une odeur de bois de merisier qui investit totalement l'espace. Les petits paysans du roman Fillette slovaque, écrit par le Suisse francophone William Ritter, ne savent pas écrire, et

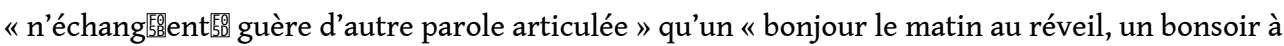
la fin de la journée » mais ils se comprennent mieux que s'ils se parlaient. In : Ritter W., Fillette slovaque, Société du « Mercure de France », Paris, 1903, pp. 226-227.

19. RothJ., Hôtel Savoy (1924), traduit de l'allemand par Françoise Besson, Gallimard, coll. « L'Imaginaire », 1999, p. 98.

20. Ibid.,p. 95

21. Leroux G., Rouletabille chez le tsar (1913), in : Les Aventures extraordinaires de Rouletabille reporter, R. Laffont, Paris, 1988, p. 401.

22. Rappelons que Brod (1884-1968) appartient au cercle des écrivains allemands de Prague. Il est souvent cité pour son amitié avec Franz Kafka. 
23. Cette scène peut être mise en relation avec un passage du roman de Roth, La Marche de Radetzky, décrivant le personnage de Charles-Joseph von Trotta, en train de guetter, depuis la triste solitude de sa chambre d'officier, le chant mélancolique des ordonnances slaves rassemblés dans les dortoirs de la caserne et formant une communauté soudée.

24. Brod M., Ein tschechisches Dienstmädchen (Une servante tchèque, 1909), A. Juncker,BerlinStuttgart-Leipzig, p. 68. Traduction par l'auteur de cet article.

25. Ibid.,pp. 108-109.

26. Lorrain J., Très russe (1886), Éd. Hubert Julia, coll. « Modernités », Paris, 1986, pp. 73-74.

27. Ibid., p. 75.

28. Cela est encore accru lorsqu'il s'agit du nom, étranger, de l'être aimé.

29. Lorrain J., Op. cit., pp. 90-91.

30. Barthes R., L'Empire des signes (1970), Seuil, coll. « Points Essais », Paris, 2007, p. 15.

31. Mann Th., La Mort à Venise (1912), traduit de l'allemand par Philippe Jaccottet, La Bibliothèque des Arts, Lausanne, 1994, pp. 101-102. C'est l'auteur de l'article qui souligne.

32. Il conserve sa dignité en masquant derrière des prétextes fallacieux la nature coupable de cet apprentissage, preuve de sa faiblesse : «(...) il étudiait le russe, parce que, disait-il, il espérait qu'au service cela lui vaudrait certains avantages ». In : Mann Th., La Montagne magique (1924), traduit de l'allemand par Maurice Betz, in :Romans et nouvelles II, Le Livre de Poche, Classiques modernes, coll. « La Pochothèque ", 1995,p. 652.

33. Ibid., p. 715, pp. 959-960.

34. Ibid., p. 959.

35. Le servage est certes aboli en 1861, sous le règne d'Alexandre II, mais il marque durablement les esprits, et se trouve souvent associé, de façon anachronique, à l'évocation de l'Empire russe.

36. Le fait est généralement connu dans ses grandes lignes: le mot "esclave " (Sklave en allemand) serait issu du nom des Slaves, dont beaucoup furent réduits en esclavage durant le haut Moyen âge. On serait donc passé d'un nom de peuple à la désignation d'un statut social et juridique, le mot ayant été scindé progressivement en deux formes distinctes morphologiquement.

37. Leroux G., Op. cit., pp. 387-593.

38. Leroux G., Op. cit., p. 580. C'est l'auteur de l'article qui souligne.

39. Le marquis Astolphe de Custine l'avait déjà bien vu, lorsque, dans son célèbre ouvrage La Russie en 1839, il s'étonnait ironiquement de "l'imprudence » du despote : "À la place de l'empereur, je ne me contenterais pas d'interdire à mes sujets la plainte, je leur défendrais aussi le chant, qui est une plainte déguisée ; ces accents si douloureux sont un aveu et peuvent devenir une accusation, tant il est vrai que, sous le despotisme, les arts eux-mêmes, lorsqu'ils sont nationaux, ne sauraient passer pour innocents; ce sont des protestations déguisées. » In: de Custine A., La Russie en 1839, Thesaurus Actes Sud, Paris, 2005, p. 665.

40. Mauthner Fr., Der letzte Deutsche von Blatna (1913), Allstein \& Co, Berlin-Vienne, 1913, p. 85.

41. Ce motif spectaculaire de la flambée de révolte "russe ", scandée par un chant guerrier, est abordé de façon frontale et accusatrice dans les nouvelles de Kessel. Mais il court plus généralement dans des textes antérieurs à 1917, ou évoquant des révoltes autres mais qui se trouvent associées à l'imaginaire russe de façon subtile car indirecte, ainsi par le biais d'un personnage: le Russe Souvarine dans Germinal d'Émile Zola (1885), la petite Marguerite des Somnambules de Broch (1931), dont l'identité hybride (franco-allemande) inquiète, et qui est fantasmatiquement qualifiée de "petite Tartare brune", nomination qui relève du même paradigme orientalisant que le mot slave. Voir Broch H., Les Somnambules, traduit de l'allemand par Pierre Flachat et Albert Kohn, Gallimard, coll. « L'Imaginaire », Paris, 1990, p. 546.

42. Kessel J., La Steppe rouge, Gallimard, Paris, 1923, p. 19. 


\section{AUTEUR}

\section{CÉCILE GAUTHIER}

Membre de l'équipe «Littérature et histoires » (Université Paris VIII), Associée au CIRCE

(Université Paris IV), ATER en Littérature comparée à l'Université de Picardie Jules Verne 